\title{
Therapeutischer Yoga und Āyurveda
}

\section{Behandlungsmöglichkeiten bei Koronarer Herzkrankheit}

Hedwig H. Gupta

\section{Einleitung}

Die koronare Herzkrankheit (KHK) ist die Manifestation der Arteriosklerose in den Herzkranzarterien. Durch die flusslimitierenden Stenosen kommt es zur Koronarinsuffizienz. Das führt zur Myokardischämie.

Man unterscheidet 2 Formen:

- asymptomatische KHK (stumme Ischämie)

- symptomatische KHK

Die symptomatische KHK beinhaltet das gesamte Spektrum von der vorübergehenden Angina pectoris über die ischämische Herzmuskelschädigung mit Linksherzinsuffizienz oder Herzrhythmusstörungen bis hin zum Herzinfarkt oder plötzlichen Herztod.

Die KHK ist immer noch die häufigste Todesursache in den Industrienationen mit einer Lebenszeitprävalenz in Deutschland für Männer 30\% und Frauen 15\%.

Die KHK ist wie wenige andere Erkrankungen mit eindeutig definierbaren Risikofaktoren assoziiert. Die Hochrisikofaktoren sind

- Zigarettenrauchen (verstärkt durch

Einnahme der Pille)

- arterielle Hypertonie

- LDL-Cholesterin-Erhöhung

- HDL-Cholesterin-Erniedrigung

- Lebensalter ( $\mathrm{m}$ : > 45, w: > 55 Lebensjahre)

- KHK/Herzinfarkt bei erstgradigen Verwandten (v.a. in jüngeren Jahren)

- Diabetes mellitus

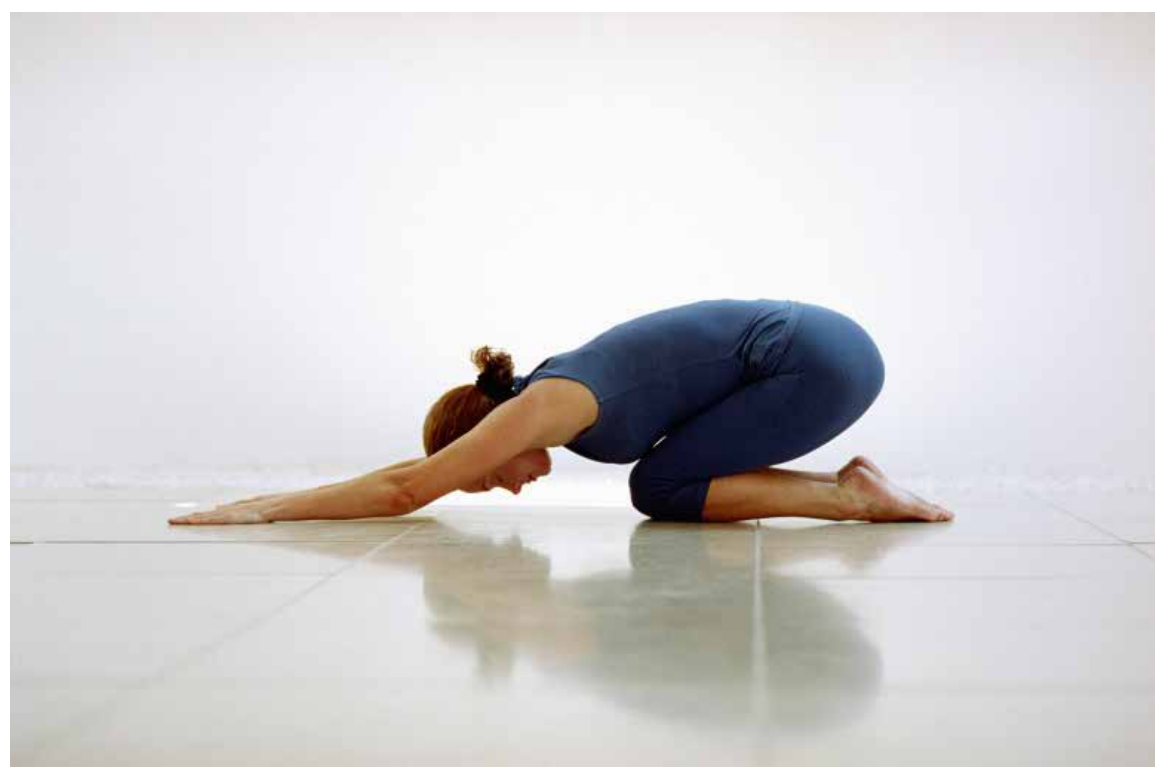

Abb. $1 \odot$ imagesource; nachgestellte Situation

An anderen Risikofaktoren sind u.a. bekannt die atherogene Diät, Adipositas, körperliche Inaktivität, andere Lipidstoffwechselstörungen, Glukosetoleranzstörungen, Entzündungszustände, Thromboseneigung, Hyperhomozysteinämie und Hyperfibrinogenämie.

\section{KHK aus āyurvedischer Sicht}

Im Āyurveda werden Herzerkrankungen als eine Entität mit verschiedenen Ausprägungen beschrieben. Herzerkrankungen werden hrdroga genannt. Sie werden von allen klassischen Autoren beschrieben [2, $3,4,5]$.
Als ursächlich werden Ernährungsfehler mit übermäßiger Einnahme von heißer, schwerer, trockener, bitterer und adstringenter Nahrung, mit häufigem und exzessivem Überessen sowie der Einnahme von Nahrung mit gegensätzlichen Eigenschaften genannt. Störungen des Stoffwechsels (agni) sind eine weitere Ursache.

Eine zu intensive Reinigungstherapie und das Unterdrücken von natürlichen Bedürfnissen, wie z.B. dem zu erbrechen, werden als weitere verursachende Faktoren beschrieben. Auch psychosomatische Aspekte wie Angst, Sorgen, Kummer oder geistige Anspannung und die Überlastung des Systems durch übermäßige körperliche Anstrengung sind 
Die koronare Herzkrankheit ist eine lebensstilassoziierte Erkrankung, die in den Industrienationen die Hauptursache für Todesfälle ausmacht.

Āyurveda und Yoga haben ein eigenes Verständnis der Pathogenese und Ätiologie, das aber wie in der westlichen Medizin die Bedeutung der Verhaltens- und Ernährungsweise anerkennt. Um die Erkrankung zu behandeln, muss der Patient seine Lebensweise in allen Aspekten nachhaltig ändern, insbesondere aber die Ernährung. Mit den Methoden des Yoga kann die notwendige Umstellung der entscheidenden Lebensstilaspekte gut unterstützt werden.

Schlüsselwörter

Koronare Herzkrankheit, KHK, āyurvedische Therapie, Yoga.

\section{ABSTRACT}

The coronary heart disease is a lifestyle-related disease, which is the main cause of death in industrialized nations.

Āyurveda and Yoga have their own conception of pathogenesis and etiology, but like Western medicine, they acknowledge the importance of behavior pattern and nutrition. In order to treat the disease, the patient has to change his way of living sustainably, especially his nutrition. The methods of Yoga are well suited to support the necessary change of the crucial aspects of lifestyle.

Keywords

Coronary heart disease, CHD, ayurvedic therapy, Yoga. mögliche ätiologische Faktoren der Herzerkrankungen.

Aus āyurvedischer Sicht werden durch diese ursächlichen Faktoren alle 3 doșas gereizt. Sie schwächen die Verdauungsfeuer (agni). Dadurch, dass die agnis dann unvollständig verbrennen, entsteht āma. Das sind Stoffwechselschlacken mit adhäsivem Charakter, die an den Gefäßwänden anhaften können. Dieses āma reizt seinerseits rasa-dhātu, die klaren Flüssigkeiten im Körper, das durch das Herz zieht. Damit wird eine Verletzung im Herzen und seinen Gefäßen hervorgerufen.

Schon deutlich bevor die ausgeprägten Symptome der Herzkrankheit auftreten, sind im Āyurveda die Vorzeichen, pūrvarūpa, beschrieben. Diese zeigen sich mit Palpitationen, Schwere in der Brust, ersten Schmerzen im Brustraum und Atemschwierigkeiten.

Hat sich die Erkrankung im Herz festgesetzt, zeigt sie sich anfangs mit einer leichten Zyanose oder anderen Verfärbungen im Gesicht, kurzen Synkopen und mildem Fieber, später mit Husten, Schluckauf und massiver Dyspnoe sowie Durst und Verdauungsstörungen, im späten Stadium mit Erbrechen, Husten mit Expektoration und dramatischen Schmerzen in der Herzgegend.

Je nach Dominanz der gereizten doșas werden unterschiedliche Formen unterschieden.
Bei Dominanz des dosa vāta stehen Tremor, Synkopen, kardiale Arrhythmien und verschiedene Schmerzformen im Herzen im Vordergrund. Die Störung kommt plötzlich auf und führt zu Dyspnoe und Schlafmangel. Die Symptome werden verstärkt nach der Verdauung von Mahlzeiten.

Bei Dominanz des doṣas pitta treten ein bitterer Geschmack, Sodbrennen, Schwitzen und brennende Schmerzen mit Erbrechen, Schwindel und Synkopen auf. Die Symptome sind während der Verdauung des Essens maximal.

Bei Dominanz des dosa kapha ist der Patient sehr müde, fühlt sich schwer und leidet an einem Gefühl von Schwere und Enge im Herzen, Druck auf der Brust und Kälte. Der Patient speichelt, ist schwindelig und hustet. Er hat keinen Appetit und einen süßen Geschmack im Mund.

Insgesamt wird die KHK als eine besonders gefährliche Erkrankung angesehen, weil sie eine Verletzung eines der zentralen Sitze von prāṇa, der Lebenskraft, beinhaltet. Das Herz ist aus vedischer Sicht nicht nur die Kreislaufpumpe, sondern auch der Sitz von subtilen Aspekten wie dem Geist und der Seele und der vitalen Anteile des Immunsystem, dem para-ojas. Jeder doṣa hat mindestens eine Unterform, die im Herzen ihren Sitz hat. Daher sind Herzerkrankungen extrem schwächende Erkrankungen!

\section{Therapie der KHK}

Für die Schulmedizin ist das Ausschalten der Risikofaktoren ein Anliegen. Dazu erfolgt die Optimierung von Cholesterin-, Blutdruck- und Blutzuckerwerten. Weiter wären die Lebensstil- und Ernährungsveränderung sowie Stressvermeidung notwendig. Diese durchzusetzen gestaltet sich jedoch im praktischen Alltag oft schwierig.

Symptomatisch bleibt beim akuten Koronarsyndrom die Intensivtherapie unersetzbar. Bei dem stabilen Koronarsyndrom wird eine Basistherapie zur Senkung der Letalität eingesetzt, z. B. mit niedrig dosierter Acetylsalicylsäure und Beta-Blockern.

Außerdem erfolgt eine antianginöse Therapie, z.B. mit Nitraten oder Kalziumantagonisten. Im Notfall existieren zudem die Möglichkeiten der Revaskularisation, der Schmerzbehandlung sowie zuletzt der Herztransplantation.

\section{Yogatherapie der KHK}

\section{Therapieziele}

Im Yoga ist das erste Ziel das Verhindern der Ursachen der Erkrankung. Die Ursachen liegen aus schulmedizinischer wie aus āyurvedischer Sicht primär in der Ernährung wie im Lebensstil des Patienten. Diese effektiv zu vermeiden, ist das primäre Ziel der Yogatherapie.

Aus āyurvedischer Sicht sind die in der Schulmedizin statistisch erwiesenen Risikofaktoren entweder geeignet, die doșas vāta (z. B. durch Nikotingenuss oder Hypertonie) und kapha (z.B. durch fettreiches Essen und Bewegungsmangel) $\mathrm{zu}$ steigern oder sie sind bereits Zeichen der Störung der Verdauungsfeuer und der Entstehung von āma, intrinsischen Schlacken (z.B. Hypercholesterinämie oder Diabetes mellitus).

Das nächste Behandlungsziel der Yogatherapie ist daher das Reinigen von agni und damit das Verbrennen von āma, den metabolischen Schlacken, die an den Gefäßen anhaften. Wenn agni sauber brennt, können schon vorhandene Schlacken durch die Verbrennung aus dem System entfernt werden und neue Schlacken werden nicht mehr produziert. 
Weiter bezweckt der Yoga stets, die betroffene Person zu stärken. Aus āyurvedischer Sicht hat jeder Mensch seine einmalige, schon bei der Geburt feststehende Konstitution, die seine besonderen Neigungen und Begabungen bestimmt. Diese zu stärken und zu stabilisieren ist stets ein wichtiges Ziel der Yogatherapie. Je besser ein Patient in seiner Konstitution gestärkt ist, desto ausgeglichener sind seine doṣas und umso größer seine Resistenz gegenüber Erkrankungen sowie die Kraft, mit der er Krankheiten überwinden kann.

Erdung ist das nächste Behandlungsziel in der Yogatherapie. Eine lebensgefährliche Erkrankung zu haben, bei der einem die Lebenskraft abgedrückt wird, bedeutet immer die Entstehung von Angst und die Schwächung des Individuums. Die Angst selbst ist aber wieder ein ursächlicher Faktor, der die Erkrankung vorantreiben kann. Ein geerdeter Mensch hingegen hat Lebensmut, er überwindet Krankheiten und Angst.

Ein weiteres, wichtiges Ziel der Yogatherapie ist die Stabilisierung des Herzens und zwar nicht nur auf körperlicher Ebene, sondern auch auf subtiler.

Das Herz ist aus yogischer Sicht nicht nur Kreislauforgan und Sitz von Geist und Seele, sondern auch noch der Spiegel des vierten und zentralen cakras, des anāhata-cakras. Dieses steuert aus Sicht der subtilen Physiologie den Tastsinn, das Tatorgan der Hände und steht in engstem Zusammenhang mit dem vijñānamayakośa, der Hülle der Intuition und mit prāṇa-vāyu, der Form von vāta-doṣa, der die Lebenskraft kontrolliert. Eine Erkrankung des physischen Herzens führt typischerweise zu einer Störung des subtilen Funktionierens, die wir in der Yogatherapie ebenso stabilisieren wollen wie die grobstoffliche Ebene. Das Erreichen all dieser Ziele davor führt zum Erreichen des letzten Ziels der Yogatherapie, nämlich den Aufbau von ojas, der Immunität. Dieses funktioniert aus āyurvedischer Sicht so, dass es die doșas so stabilisiert, dass diese nicht wieder aus dem Gleichgewicht fallen und den Patienten erkranken können. Je mehr ojas aufgebaut ist, desto höher die Krankheitsresistenz des Patienten.
Techniken und Vorgehensweise

Abhängig vom Stadium der KHK beginnt man bei schweren Ausprägungen zunächst nur mit subtilen Yogatechniken. Dazu zählen prānāāāma-Übungen, wobei einfache Techniken wie pūrṇa-prāṇāyāma, die yogischen Vollatmung, oder nāḍ̄i -śodhana-prāṇāyāma, die Wechselatmung anfangs im Vordergrund stehen.

prāṇāyāma-Übungen sind ausgesprochen wichtige Instrumente der Yogatherapie bei Herzerkrankungen, weil sie in ihrer körperlichen Belastung sehr gut steuerbar sind, direkt auf der Ebene von prāṇa-vāyu wirken und je nach Art und Ausprägung entweder reinigend oder aber stabilisierend sein können.

Eher ruhige, ausatembetonte Atemtechniken stabilisieren und erden, gleichen die doșas aus und entlasten das Herz. Eher schnelle, aktivierende Atemtechniken dagegen fördern agni und die Verbrennung von āma, fordern das Herz-Kreislauf-System und stärken den Patienten. Abhängig von der Belastbarkeit des Patienten und dem Stadium der KHK sind also unterschiedliche Formen von prānāyāma indiziert.

Andere auch bei starker körperlicher Einschränkung einsetzbare Techniken sind mantras (Klänge), Visualisierungs- und Selbstwahrnehmungsübungen. All diese Techniken wirken herzstabilisierend und gleichen die doṣas aus. Sie führen nach innen und fördern die Selbstwahrnehmung.

An körperlichen Übungen empfehle ich, bei fortgeschrittener KHK zunächst mit einfacher Gehmeditation zu beginnen: langsames, bewusstes Gehen mit Wahrnehmung der Füße am Boden. Auch solche banalen Übungen helfen dem Patienten, den Halt wiederzugewinnen und Selbstvertrauen und -achtsamkeit zu entwickeln.

Dazu kann anfangs bei starker Herzschwäche die Praxis von yogāsanas als Stuhl-Yoga erfolgen. Dabei müssen noch nicht gleich komplette āsanas geübt werden, sondern es können z. B. Aspekte der pavanamūktāsana-Serien oder anderen vereinfachten āsana-Folgen eingesetzt werden. Es geht darum, dass die Patienten zunehmend wieder ein Körpergefühl entwickeln, die eigenen Fähigkeiten wieder spüren und langsam die eigene Leistungs-

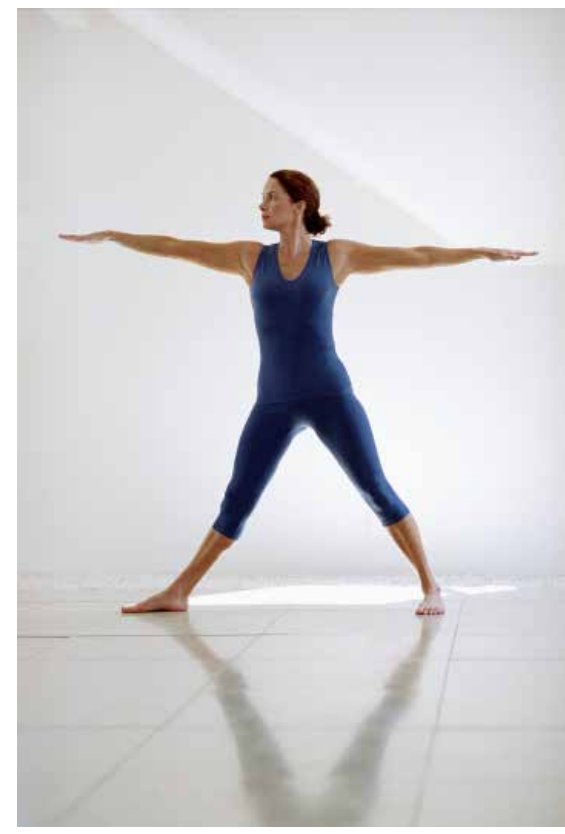

Abb. 2 @ imagesource; nachgestellte Situation

fähigkeit und deren Grenzen wahrnehmen können. Im Verlauf der Rehabilitation ist eine langsame Steigerung möglich, mit Einbeziehen von āsanas im Liegen und Stehen oder Dreh-āsanas (Abb. 2).

Eine für den Herzpatienten interessante Form des Yoga ist auch das restaurative Yoga, bei dem Haltungen passiv eingenommen werden. Der Patient wird einer veränderten Wirkung der Schwerkraft ausgesetzt, was zum Umverteilen der passiven Blutvolumina im Sinne eines Kreislauftrainings, zur Dehnung und Öffnung und zum angstfreien Spüren des Körpers eingesetzt werden kann.

Mit zunehmender Kreislaufstabilität ist das langsame Einführen von Umkehrhaltungen, z. B. mit viparita-karan $i$ indiziert.

Bei stabilen Herz-Kreislauf-Verhältnissen und sicherer Selbstwahrnehmung kann der Patient vorsichtig auch an einem allgemeinen Yoga-Übungsprogramm teilnehmen.

\section{Āyurvedische Therapie der KHK}

Das Verständnis von Ätiologie und Pathogenese der KHK übernimmt der therapeutische Yoga aus dem Āyurveda, dem vedischen Medizinsystem. Daher sind auch die Ziele der Behandlung analog. Auch im Āyurveda ist das erste Ziel das nidāna- 
parivārjana, das Verhindern der Ursache. Um agni zu stärken und āma zu verdauen, empfiehlt man im Āyurveda, Ghī zum Kochen zu verwenden, das Trinken von Ingwerwasser oder Wasser mit LaghuPañcamūla (einer entzündungshemmenden Kombination von 5 Wurzeln). In āma-Stadien sollte der Patient Milchprodukte und Fleisch von Meerestieren vermeiden. Für den Herzpatienten wird eine reine, leichte, nährende Ernährung, hinreichend Ruhe, leichte körperliche Betätigung und langsames Gehen, morgens gerne im feuchten Gras, empfohlen. Kühlende Cremes und Auflagen werden über der Herzgegend empfohlen. Herzpatienten sollten alles vermeiden, was zu Spannung, übermäßiger Belastung, Fehlverdauung und Verstopfung führt.

An dosa-beruhigender Therapie werden innerlich Heilpflanzen aus unterschiedlichen Wirkgruppen empfohlen. Dazu gehören v.a. hrdaya, herzstärkende Pflanzen. Diese fördern zudem das ojas, kühlen und beruhigen vāta-dosa. Arjuna (Terminalia arjuna) oder Tarūṇī (Rosa centifolia, Rose) sind typische Vertreter dieser Kräutergruppe.

Bei eher niedrigem Blutdruck und Herzschwäche werden hrdottejaka, herzaktivierende Pflanzen eingesetzt. Diese aktivieren das Herz, schneller und stärker zu schlagen. Sie sind eher erhitzend. Dazu gehören z. B. Karpūra (Cinnamomum camphora, Kampfer) oder Marica (Piper nigrum, Schwarzer Pfeffer). Außerdem kommen bei der KHK Pflanzen zum Einsatz, die die Durchblutung der Gefäße fördern, die Gruppe der rakta-srāvaka-dravya. $\mathrm{Zu}$ ihr gehören u.a. Vāca (Acorus calamus, Kalmuswurzel) oder Guggulu (Commiphora mukul, indische Myrrhe).

$\mathrm{Zu}$ Stärkung des Patienten werden rasāyana-dravya, regenerierende Substanzen, gegeben. Dazu kommen etwa Balātailam oder Brahmarasāyana infrage. In der äußerlichen Therapie werden Auflagen für das Herz mit herzstärkenden Heilkräutern oder leicht erwärmende lokale Ölbehandlungen wie hrdbasti, hrdpicu etc. indiziert. Ist die Erkrankung langfristig vorhanden, aber der Patient nicht sehr geschwächt, werden auch doṣa-reinigende Techniken des Pañcakarma eingesetzt. Gerade bei der KHK gehört dies aber in die
Hand eines sehr erfahrenen Āyurveda-Arztes, weil Pañcakarma-Therapien reduzierend wirken und daher im Falle einer KHK mit größter Sorgfalt durchzuführen sind.

\section{Studien zur Yogatherapie bei KHK}

Seit Jahrzehnten wird zur Yogatherapie bei KHK geforscht.

Der älteste der Autorin bekannte wissenschaftliche Bericht über den positiven Effekt prānāyāma-artiger Atemtechniken bei KHK ist aus dem Jahre 1948. Es handelt sich um eine Sammlung von 11 Fallbeschreibungen von Patienten mit Angina pectoris, einige mit Risikofaktoren wie Hypertension, verminderter Glukosetoleranz, andere im Zustand nach Herzinfarkt, bei denen allen die Symptome durch „aufmerksame Atmung“ verringert wurden [6].
Viele Arbeiten beschäftigen sich mit der Wirkung von Yoga auf kardiovaskuläre Risikofaktoren. Durch yogische Techniken

- sinkt der Blutdruck bei Hypertonikern $[7,8,9,10,11,12,13,14,22,23]$,

- vermindert sich die Herzfrequenz [22],

- sinkt der Serumcholesterinspiegel auf Normwerte [7, 15, 16, 17, 21, 23],

normalisieren sich Glukosemetabolismus [16] und Gewicht [22, 23],

- werden Stress und Nervosität abgebaut $[18,19,20]$,

- Ängstlichkeit reduziert [21] und

- wird das Abgewöhnen von Rauchen und Alkohol erleichtert [14, 20].

Es wurde sogar mittels Angiografie nachgewiesen, dass sich durch Yogapraxis die Durchblutung der Koronarien verbessert und das Voranschreiten der Erkrankung im Vergleich zu einer Kontrollgruppe verzögert wird [21]. 
In keiner der Studien wurden unerwünschte Wirkungen beobachtet.

\section{Zusammenfassende Beurteilung}

Die koronare Herzerkrankung ist eine lebensstilassoziierte Erkrankung, die in den Industrienationen die Hauptursache für Todesfälle ausmacht.

Âyurveda und Yoga haben ein eigenes Verständnis der Pathogenese und Ätiologie, das aber ebenfalls die Bedeutung der Verhaltens- und Ernährungsweise anerkennt. Um eine solche Erkrankung zu behandeln, muss der Patient seine Lebensweise in allen Aspekten nachhaltig ändern, insbesondere aber die Ernährung. Die Beratung, die den Patienten im Allgemeinen gegeben wird, spricht sie auf intellektueller Ebene an. Die Patienten sollen verstehen, aus welchem Grund sie etwas ändern sollen. Verhalten und gerade die Ernährung sind höchst emotional gesteuerte Aspekte des Lebens. Diese willentlich zu ändern, braucht eine geistig emotionale Disziplin. Mit den Methoden des Yoga kann die Lebensweise gut behandelt werden. Die Wirksamkeit dieser Techniken spiegelt sich in vielen wissenschaftlichen Untersuchungen.

Der Āyurveda nutzt diese Effekte und gibt genaue Konzepte, wie mit Phytopharmaka, Ernährung und Reinigungstechniken das Leben der KHK-Patienten gesichert werden kann.

Interessenkonflikte: Die Autorin erklärt, dass keine wirtschaftlichen oder persönlichen Verbindungen bestehen.

\section{Online zu finden unter:}

http://dx.doi.org//10.1055/s-0041-102474

\section{Literatur}

[1] Herold G. Innere Medizin. München: Elsevier; 2006

[2] Carakasamhitā Cikitsāsthāna, Kapitel 26

[3] Suśrutasamhitā. Uttaratantra, Kapitel 43

[4] Aśtāniganahṛdayam Cikitsāsthāna, Kapitel 6

[5] Mādhavanidānam, Kapitel 29
[6] Friedell A. Automatic attentive breathing in angina pectoris. Minn. Med. 1948; 31: 875888

[7] Jain MD, Tiwari R, Shankaranand D. Comparative clinical study of combined holistic medical approach with medicinal monotherapy \& placebo on 121 male patients with mild high blood pressure and blood cholesterol. Clinical Experience with Yoga \& Medicine, part II, Holistic Society of India; 1993: 18-22

[8] Patel C. Randomized controlled trial of Yoga and biofeedback in management of hypertension". Lancet ii 1975; 93-95

[9] Patel C. Yoga and biofeedback in the management of hypertension. Lancet ii 1973; 1053-1055

[10] Silverberg DS. Non-pharmacological treatment of hypertension. J Hypertens 1990; 8: 521-526

[11] Schneider RH, Staggers F, Alexander CN et al. A randomized controlled trial of stress reduction for hypertension in older African Americans. Hypertension 1995; 26: 820827

[12] Alexander CN, Schneider RH, Staggers F et al. Trial of stress reduction for hypertension in older African Americans II. sex and risk subgroup analysis. Hypertension 1996; 28: $228-237$

[13] Patel C. 12-month follow-up of yoga and biofeedback in the management of hypertension. Lancet I 1975; 62-64

[14] Patel C, Marmot MG, Terry D] et al. Trial of relaxation in reducing coronary risk: four year follow up". Br Med J 1985; 290: 11031106

[15] Jevning R, Wallace RK, Beidebach M. The physiology of meditation: A review. A wakeful hypometabolic integrated response. Neurosci Biobehav Rev 1992; 16: 415-424

[16] Cooper MJ, Aygen MM. A relaxation technique in the management of hypercholesterolemia. J Human Stress 1979; 5: 2427

[17] Jain SC, Uppal A, Bhatnagar SOD et al. A study of response pattern of non-insulin dependent diabetics to yoga therapy". Diabetes Res Clin Pract 1993; 19: 69-74

[18] Udapa KN, Singh RH, Settiwar RM. Studies on physiological, endocrine, and metabolic response to the practice of yoga in young normal volunteers. J Res Ind Med 1993; 6: 345-353

[19] Schell F], Allolio B, Schonecke OW. Physiological and psychological effects of hathayoga exercise in healthy women. Int J Psychosom 1994; 41: 46-52
[20] West MA. Physiological effects of meditation: A longitudinal study. Br Med J Soc Clin Psychol 1994; 18: 219-226

[21] Yogendra J, Yogendra H], Ambardekar S et al. Beneficial effects of yoga lifestyle on reversibility of ischaemic heart disease caring heart project of International Board of Yoga. J Assoc Physicians India 2004; 52: 283-289

[22] Pal A, Srivastava N, Narrain VS et al. Effect of yogic intervention on the autonomic nervous system in the patients with coronary artery disease: a randomized controlled trial. East Mediterr Health J 2013; 19 : 452-458

[23] Pal A, Srivastava N, Tiwari S et al. Effect of yogic practices on lipid profile and body fat composition in patients of coronary artery disease. Complement Ther Med 2011; 3: 122-127

\section{ÜBER DIE AUTORIN}

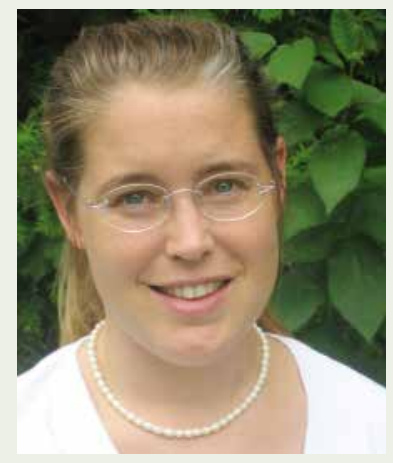

Hedwig H. Gupta, nach dem Studium der Humanmedizin Postgraduiertenstudium von Ayurveda, Yoga und Sanskrit an der BHU, Indien. Autorin von Fachbüchern und Fachartikeln. Leiterin der Vidya-Sagar-Akademie für Ayurveda und Yogatherapie. Mitbegründerin der DÄGAM (Deutsche Ärztegesellschaft für Ayurveda-Medizin) und der DeGYT (Deutsche Gesellschaft für Yogatherapie).

KORRESPONDENZADRESSE

Dr. med. Hedwig H. Gupta

Fachärztin für Orthopädie, Schwerpunkt

Rheumatologie

Āyurveda, therapeutischer Yoga, Akupunktur, Manuelle Medizin

Seestr. 5

71638 Ludwigsburg

www.dr-gupta.de

www.vidya-sagar.de

E-Mail: info@dr-gupta.de 